OPTIMAL REPLACEMENT POLICIES FOR SYSTEMS WITH MULTIPLE STANDBY COMPONENTS

\author{
Hyo-Seong Lee \\ Department of Industrial Engineering \\ Kyung Hee University, Kiheung, Yongin-goon \\ Kyunggi-do, 449-900, Korea \\ Mandyam M. Srinivasan \\ Department of \\ Industrial \& Operations Engineering \\ University of Michigan \\ Ann Arbor, MI 48109-2117
}

Technical Report 90-31

November 1990 


\title{
Optimal Replacement Policies \\ for Systems with Multiple Standby Components
}

\author{
Hyo-Seong Lee \\ Department of Industrial Engineering \\ Kyung Hee University, Kiheung, Yongin-goon \\ Kyunggi-do, 449-900, Korea \\ Mandyam M. Srinivasan \\ Department of Industrial \& Operations Engineering, \\ The University of Michigan, \\ Ann Arbor, MI 48109-2117, U.S.A.
}

\begin{abstract}
We consider preventive replacement policies for a standby system which operates with $\mathrm{N}$ components. The failure rate of the operating component is assumed to be constant, and standby components are not subject to failure. The system is inspected at different points in time in order to determine whether it is to be replaced or not. At each inspection point, a decision is made on whether to replace the operating system with a new system. The decision is based on the number of components that have failed in the system, at the time of inspection. If this number exceeds a threshold value, $r$, the system is replaced. Otherwise it continues to be used at least until the point in time at which it is next inspected. The time interval between two successive inspection points is a random variable which follows an arbitrary distribution.

We consider two replacement options if the complete system fails during operation: (i) replace the system at the time an inspection reveals that the system has failed, and (ii) replace the system the instant it fails. For both options, we first obtain an expression for the expected cost incurred per unit time for a given threshold value, under a certain cost structure. We next prove that there is an optimal threshold value, using a characteristic of the cost function, and develop a simple yet efficient procedure to find this optimal threshold value.
\end{abstract}




\section{Introduction}

Preventive replacement policies for systems that are subject to stochastic failures have been studied extensively in the past. Such policies are typically preferred if the failure of the system during its operation is costly or dangerous (Barlow and Proschan 1965). An excellent survey of the work done in this area is given in Sherif and Smith (1981). Most of the previous studies, however, treat models for single- or two-component systems. Only a limited studies have been done on replacement policies for systems consisting of more than two components (see, for example, the papers by Meine and Asakura (1969), and Gertsbakh and Popov (1972).

In this paper, we present a preventive replacement policy for a standby system with $\mathrm{N}$ components in which only one component is in operation at a time, while the others are in a standby condition. If the component in operation fails, one of the standby components is immediately switched into operation and starts to work. It is assumed that the failure rate is the same constant for each component while it is in operation, and that standby components are not subject to failure. If all the $\mathrm{N}$ components fail, this is considered as a system failure.

We assume that the system is not easily accessible for inspection, and hence the state of the system (number of failed components) can be identified only through inspections which are made at certain points in time. The time interval between two inspections is assumed to be an independent random variable following an arbitrary distribution. The decision on whether the system is to be replaced at the time the inspection is made, is based on the number of components in the system that have failed up to that point in time.

Thus, the operating characteristics of the replacement policy are as follows: If the total number of failed components at an inspection point is less than a certain prespecified value $r(r \leq N)$, the system continues to operate at least until the next inspection is made. On the other hand, if the total number of failed components at an inspection point is greater than or equal to $r$, the system is immediately replaced with a new one. It is assumed that the time for inspections and/or replacements is negligible.

The following costs are considered : i) a cost $\mathrm{C}_{\mathrm{f}}$ which is incurred when a failed system is replaced; in addition to the replacement cost, this cost could include other costs that may result from the failure of the system except for the system down-time cost which is considered separately; ii) a cost $C_{p}$ which is incurred when a system is replaced before failure $\left(C_{p}<C_{f}\right)$; iii) a system down-time cost of $C_{d}$ which is incurred per unit time during the time that the system is 
down, if a failure occurs during operation (between two inspections), and the system is not replaced until the next inspection point.

Let $\mathrm{TC}(\mathrm{r}, \mathrm{N})$ denote the expected cost per unit time when $\mathrm{r}$ is used as a threshold value and the system has $\mathrm{N}$ components. The objective of this paper is to determine the optimal threshold value $r$ which balances the associated costs and results in a minimum total long-run average cost per unit time, i.e., $\min _{0 \leq r \leq N} C(r, N)$.

It should be noted that although there is a definite difference in the cost structure, this problem has an analogy with $(\mathrm{s}, \mathrm{S})$ production/inventory problems and threshold-value type queueing control problems. See, for instance, Heyman(1968), Lee and Srinivasan(1989), Srinivasan and Lee(1991) and Gavish and Graves(1980), among others. In the following sections, first an expression for the expected cost per unit time for a given control value will be derived and following that some properties of the cost functions are investigated. Based on these properties an efficient procedure to find the optimal control value $r^{*}$ is developed.

We first consider the situation where the failed system is replaced only when an inspection reveals the failure. In a subsequent section, we consider the situation in which a failed system is replaced with a new one at the instant it fails.

\section{The Analysis}

\subsection{General Approach}

Suppose that the failures of components are due to the system being subject to shocks, that is, every time a shock occurs, the component in operation fails. Let $\lambda$ denote the rate at which the shocks occur. The shocks are assumed to arrive according to a Poisson process. The time between two inspections is assumed to be an independent random variable, $\mathrm{V}$, following a general distribution $\mathrm{G}$.

The time interval between two successive replacements is termed a cycle. Let $C(r, N)$ denote the expected cost incurred during a cycle and let $\mathrm{L}(\mathrm{r})$ denote the expected length of a cycle, when $\mathrm{r}$ is used as a threshold value and the number of components in the system is $\mathrm{N}$. Then from the renewal reward theorem (see, for example, Ross 1974), the expected cost per unit time is expressed as

$$
\mathrm{TC}(\mathrm{r}, \mathrm{N})=\frac{\mathrm{C}(\mathrm{r}, \mathrm{N})}{\mathrm{L}(\mathrm{r})}
$$


Let $P_{f}(r, N)\left(P_{p}(r, N)\right)$ denote the long-run fraction of times that replacements are made as a result of system failures (replacements are made as a preventive measure) are made when $r$ is used as a threshold value and the number of components is $\mathrm{N}$. Also let $\tau(\mathrm{r}, \mathrm{N})$ denote the expected length of the system down-time in a cycle. Then $\mathrm{C}(\mathrm{r}, \mathrm{N})$ is expressed as

$$
\mathrm{C}(r, N)=\mathrm{C}_{\mathrm{p}} \mathrm{P}_{\mathrm{p}}(\mathrm{r}, \mathrm{N})+\mathrm{C}_{\mathrm{f}} \mathrm{P}_{\mathrm{f}}(\mathrm{r}, \mathrm{N})+\mathrm{C}_{\mathrm{d}} \tau(\mathrm{r}, \mathrm{N}) .
$$

Noting that $P_{p}(r, N)=1-P_{f}(r, N)$, from equations (2.1) and (2.2) we obtain

$$
T C(r, N)=\frac{C_{p}+\left(C_{f}-C_{p}\right) P_{f}(r, N)+C_{d} \tau(r, N)}{L(r)} .
$$

\subsection{Computing the term $P_{f}(r, N)$}

Let $\mathrm{q}_{\mathrm{j}}$ denote the probability that there are $\mathrm{j}$ shocks during an inspection interval, i.e.,

$$
q_{j}=\int_{0}^{\infty} \frac{(\lambda t) j}{j !} e^{-\lambda t} d G(t) .
$$

Note that $\mathrm{q}_{\mathrm{j}}, \mathrm{j}=1, \ldots, \mathrm{N}-1$, is the probability that $\mathrm{j}<\mathrm{N}$ components fail during an inspection interval. To obtain $P_{f}(r, N)$, we condition on the number of shocks during the first inspection interval. With probability $q_{j}, j$ shocks occur during the first inspection interval. If $j \geq N$, the system fails, and if $\mathrm{r} \leq \mathrm{j} \leq \mathrm{N}-1$, a preventive replacement is made at the first inspection point.

If $j<r$, then the number of failed components at the first inspection point is less than $r$. Hence the system will not be replaced at least until the next inspection takes place, at which point the probability that the system will be replaced due to a failure is just $P_{f}(r-j, N-j)$. Thus, we obtain:

$$
P_{f}(r, N)=\sum_{j=N}^{\infty} q_{j}+\sum_{j=0}^{r-1} q_{j} P_{f}(r-j, N-j) .
$$

Collecting terms involving $P_{f}(r, N)$, we obtain the following recursive equation:

$$
P_{f}(r, N)=\frac{1}{1-q_{0}}\left\{\sum_{j=N}^{\infty} q_{j}+\sum_{j=1}^{r-1} q_{j} P_{f}(r-j, N-j)\right\}
$$

where, by definition, $P_{f}(0, k)=0$ for all $k \geq 0$. As an initial value for equation (2.6), we use

$$
P_{f}(1, k)=\frac{1}{1-q_{0}} \sum_{j=k}^{\infty} q_{j}, \quad \text { for } k \geq 1 .
$$




\subsection{Computing the term $\tau(r, N)$}

Let $V_{i}$ denote the length of an inspection interval given that $i$ shocks arrived during that inspection interval and let $\bar{v}_{i}$ denote its expected value. We express $\bar{v}_{i}$, using Bayes' formula, as

$$
\bar{v}_{i}=E\left(V_{i}\right)=\frac{1}{q_{i}} \int_{0}^{\infty} \frac{(\lambda t)^{i}}{i !} e^{-\lambda t} t d G(t)
$$

After some algebraic manipulation of equation (2.8), we get

$$
\bar{v}_{i}=\frac{(i+1) q_{i+1}}{\lambda q_{i}} \text {. }
$$

We now obtain the expression for $\tau(\mathrm{r}, \mathrm{N})$ by conditioning on the number of shocks which occur during the first inspection interval as

$$
\tau(r, N)=\sum_{j=N}^{\infty} q_{j} \frac{j+1-N}{j+1} \bar{v}_{j}+\sum_{j=0}^{r-1} q_{j} \tau(r-j, N-j) .
$$

Equation (2.10) is intuitively explained as follows. With probability $\mathrm{q}_{\mathrm{j}}$, there are $\mathrm{j}$ shocks during the first inspection interval. If $j$ is less than $r$, then there will be no action at the first inspection point and the system is not replaced at least until the next inspection point. In this case, the expected length of the system down-time is $\tau(\mathrm{r}-\mathrm{j}, \mathrm{N}-\mathrm{j})$, which is the second term in equation (2.10). If $\mathrm{j}$ lies between $\mathrm{r}$ and $\mathrm{N}-1$, a preventive replacement is made at the end of the first inspection and there is no system down-time. On the other hand, if $\mathrm{j}$ is greater than or equal to $\mathrm{N}$, the system fails the moment the $\mathrm{N}^{\text {th }}$ shock occurs, and the system is down from this point onwards until the end of the first inspection interval. The expected length of the system down-time in this case is $\frac{j+1-N}{j+1} \bar{v}_{j}$ and this is the first term in equation (2.10). Substituting the value for $\bar{v}_{i}$, as given by equation (2.9), into equation (2.10), and collecting the terms involving $\tau(r, N)$, we obtain :

$$
\tau(r, N)=\frac{1}{1-q_{0}}\left[E(V)-\frac{1}{\lambda}\left\{\sum_{j=0}^{N} j q_{j}+N\left(1-\sum_{j=0}^{N} q_{j}\right)\right\}+\sum_{j=1}^{r-1} q_{j} \tau(r-j, N-j)\right],
$$

with $\tau(0, N)=0$. As an initial value for equation $(2.11)$, we use

$$
\tau(1, \mathrm{k})=\frac{1}{1-q_{0}}\left[E(V)-\frac{1}{\lambda}\left\{\sum_{j=0}^{k} j q_{j}+k\left(1-\sum_{j=0}^{k} q_{j}\right)\right\} .\right.
$$




\subsection{Computing the term $\mathrm{L}(\mathrm{r})$}

Observe that cycle times are independent of $\mathrm{N}$ because decisions on replacements are made only at inspection points based on the information about the number of failed components. Thus, the expected cycle time is a function of $r$ alone. The expression for the expected cycle time, $L(r)$, can be obtained by conditioning on the number of shocks which occur during the first inspection interval as:

$$
L(r)=\frac{1}{1-q_{0}}\left\{E(V)+\sum_{j=1}^{r-1} q_{j} L(r-j)\right\},
$$

with

$$
L(1)=\frac{E(V)}{1-q_{0}}, \quad \text { and } L(0)=0 \text {. }
$$

From equations (2.6), (2.11) and (2.13), we can compute $P_{f}(r, N), \tau(r, N)$ and $L(r)$ recursively starting from $r=1$. Substituting these values into equation (2.3), we obtain $T C(r, N)$, the expected cost per unit time. Once $\tau(r, N)$ and $L(r)$ are computed, we can also obtain the system availability, $\mathrm{V}(\mathrm{r}, \mathrm{N})$, as

$$
\mathrm{V}(\mathrm{r}, \mathrm{N})=1-\frac{\tau(\mathrm{r}, \mathrm{N})}{\mathrm{L}(\mathrm{r})} .
$$

The expected number of components that fail during a cycle, $\mathrm{K}_{\mathrm{f}}(\mathrm{r}, \mathrm{N})$, is similarly obtained as

$$
K_{f}(r, N)=\frac{1}{1-q_{0}}\left[\sum_{j=r}^{N} j q_{j}+N \sum_{j=N+1}^{\infty} q_{j}+\sum_{j=1}^{r-1} q_{j}\left\{j+K_{f}(r-j, N-j)\right\}\right] .
$$




\section{Finding the Optimal Control Value}

In this section, we obtain the optimal value of $r$. To that end, we first develop three properties for the functions $P_{f}(r, N), \tau(r, N)$ and $L(r)$, stated as Lemmas 1"through 3. These properties are not only used to devise the algorithm to find the optimal threshold value but they also significantly reduce the computational effort required to obtain $\mathrm{P}_{\mathrm{f}}(\mathrm{r}, \mathrm{N})$ and $\tau(\mathrm{r}, \mathrm{N})$.

Lemma 1. $L(r)>L(r-1), \quad$ for $r \geq 1$.

Proof The proof is easily obtained by induction on $r$, using equation (2.13). We omit the details.

Note that Lemma 1 implies that $\mathrm{L}(\mathrm{r})$ is an increasing function with respect to $\mathrm{r}$.

Lemma 2. Let $A(r, N)=\frac{P_{f}(r, N)-P_{f}(r-1, N)}{L(r)-L(r-1)}$, and $B(r, N)=\frac{\tau(r, N)-\tau(r-1, N)}{L(r)-L(r-1)}$. Then, for $1 \leq \mathrm{n} \leq \mathrm{r}-1$,
(a) $\mathrm{A}(\mathrm{r}, \mathrm{N})=\mathrm{A}(\mathrm{r}-\mathrm{n}, \mathrm{N}-\mathrm{n})$, and
(b) $\mathrm{B}(\mathrm{r}, \mathrm{N})=\mathrm{B}(\mathrm{r}-\mathrm{n}, \mathrm{N}-\mathrm{n})$.

Proof We prove Lemma 2a) by induction on r. Lemma 2b) is proved in a similar manner.

i) Base Step: If $r=2$, then since $L(0)=P_{f}(0, k)=0$, from equations (2.6) and (2.13), we have

$$
A(2, N)=\frac{P_{f}(2, N)-P_{f}(1, N)}{L(2)-L(1)}=\frac{P_{f}(1, N-1)-P_{f}(0, N-1)}{L(1)-L(0)}=A(1, N-1) .
$$

ii) For the induction step, suppose equation (3.2) is true for $r \leq k-1$. We now prove that it is true for $r=k$. Again, from equations (2.6) and (2.13), we have

$$
A(k, N)=\frac{P_{f}(k, N)-P_{f}(k-1, N)}{L(k)-L(k-1)}=\frac{\sum_{j=1}^{k-1} q_{j}\left\{P_{f}(k-j, N-j)-P_{f}(k-1-j, N-j)\right\}}{\sum_{j=1}^{k-1} q_{j}\{L(k-j)-L(k-1-j)\}} .
$$

From the induction hypothesis, we note that the term $\frac{P_{f}(k-j, N-j)-P_{f}(k-1-j, N-j)}{L(k-j)-L(k-1-j)}$ has the same value for $j=1, \ldots, k-1$. Since $\sum_{i=1}^{k} q_{i} b_{i} / \sum_{i=1}^{k} q_{i} a_{i}=c$ if $b_{i} / a_{i}=c$ for all $i$, we can prove that $A(k, N)=A(k-n, N-n)$, for $1 \leq n \leq k-1$. So, by the principle of mathematical induction, Lemma 2a) holds. 
In the algorithm we shall present shortly, $\mathrm{P}_{\mathrm{f}}(\mathrm{r}, \mathrm{N})$ and $\tau(\mathrm{r}, \mathrm{N})$ are calculated using Lemma 2 instead of equations (2.6) and (2.11). From Lemma 2, $A(r, N)=A(1, N-r+1)$ and $B(r, N)=B(1, N-r+1)$. Thus $P_{f}(r, N)$ and $\tau(r, N)$ are expressed recursively as

$$
\begin{aligned}
& P_{f}(r, N)=P_{f}(r-1, N)+\{L(r)-L(r-1)\} \frac{P_{f}(1, N-r+1)}{L(1)} \\
& \tau(r, N)=\tau(r-1, N)+\{L(r)-L(r-1)\} \frac{\tau(1, N-r+1)}{L(1)}
\end{aligned}
$$

Since $L(1), P_{f}(1, N-r+1)$ and $\tau(1, N-r+1)$ are all obtained very simply in closed form, to compute $\mathrm{P}_{f}(\mathrm{r}, \mathrm{N})$ and $\tau(\mathrm{r}, \mathrm{N})$, we only need to compute $\mathrm{P}_{\mathrm{f}}(\mathrm{r}-1, \mathrm{~N}), \tau(\mathrm{r}-1, \mathrm{~N})$ and $\mathrm{L}(\mathrm{r})$. Therefore, $\mathrm{L}(\mathrm{r})$ is the only term in which we must use the original recursive method, namely equation (2.13), for the calculation. This reduces the computational effort significantly. Let

$$
h(r, N)=\left(C_{f}-C_{p}\right) P_{f}(r, N)+C_{d} \tau(r, N),
$$

Lemma 3. For a given value of $\mathrm{N}$,

(a) $h(r, N)>h(r-1, N)$, and (b) $\frac{h(n, N)-h(r, N)}{L(n)-L(r)}<\frac{h(n, N)-h(r+1, N)}{L(n)-L(r+1)}, \quad N \geq n>r+1$.

Proof To prove Lemma 3, we first develop some additional properties which are stated as properties 1 through 5. Proofs of these properties are provided in the appendix.

Property 1. $\quad P_{f}(r, N)$ and $\tau(r, N)$ are increasing in $r$ for a given $N$.

Property 2. $\mathrm{P}_{\mathrm{f}}(\mathrm{r}, \mathrm{N})$ and $\tau(\mathrm{r}, \mathrm{N})$ are decreasing in $\mathrm{N}$ for a given $\mathrm{r}$.

Property 3. (a) $\mathrm{P}_{f}(\mathrm{r}, \mathrm{N})-\mathrm{P}_{\mathrm{f}}(\mathrm{r}-1, \mathrm{~N}) \leq \mathrm{P}_{\mathrm{f}}(\mathrm{r}, \mathrm{N}-1)-\mathrm{P}_{\mathrm{f}}(\mathrm{r}-1, \mathrm{~N}-1)$, and

(b) $\tau(r, N)-\tau(r-1, N) \leq \tau(r, N-1)-\tau(r-1, N-1)$.

Property 4. $A(r, N)$ and $B(r, N)$ are increasing in $r$ for a given $N$.

Property 5. $\frac{h(n, N)-h(n-1, N)}{L(n)-L(n-1)}>\frac{h(n-1, N)-h(n-2, N)}{L(n-1)-L(n-2)}>\frac{h(n-2, N)-h(n-3, N)}{L(n-2)-L(n-3)}>\cdots .$.

Using these properties, we now prove Lemma 3. From the definition of $h(r, N)$ together with the facts that both $C_{f}-C_{p}$ and $C_{d}$ are positive, $h(r, N)$ is increasing in $r$ for a given $N$ if $P_{f}(r, N)$ and 
$\tau(r, N)$ are increasing in $r$ for a given $N$. From property 1 , both $P_{f}(r, N)$ and $\tau(r, N)$ are increasing in $r$ for a given $N$, and so Lemma $3 a$ ) is true. To prove Lemma $3 b$ ), we use the following identity:

If $a, b, c, d, e, f$ are all positive, then $\frac{a}{b}>\frac{c}{d}>\frac{e}{f}$ holds if and only if $\frac{a}{b}>\frac{a+c}{b+d}>\frac{a+c+e}{b+d+f}$.

If we apply this identity to property 5 , we obtain

$$
\frac{h(n, N)-h(r, N)}{L(n)-L(r)}<\frac{h(n, N)-h(r+1, N)}{L(n)-L(r+1)}, \quad \text { for } n>r+1
$$

Note that the expected cost per unit time can be written as $\operatorname{TC}(r, N)=\frac{C_{p}+h(r, N)}{L(r)}$. Using the above Lemmas, we are now in a position to present the main result of this section, which gives an important characteristic of the cost function $\mathrm{TC}(\mathrm{r}, \mathrm{N})$. This characteristic is that, in the process of evaluating $\mathrm{TC}(\mathrm{r}, \mathrm{N}), \mathrm{r}=1, \ldots, \mathrm{N}$, the first local minimum encountered is the global minimum. This is stated below as Theorem 1 .

\section{Theorem 1.}

$$
\text { If } \mathrm{TC}(\mathrm{r}+1, \mathrm{~N})>\mathrm{TC}(\mathrm{r}, \mathrm{N}) \text {, then } \mathrm{TC}(\mathrm{n}, \mathrm{N})>\mathrm{TC}(\mathrm{r}, \mathrm{N}) \quad \text { for all } \mathrm{n}>\mathrm{r}>0 \text {. }
$$

Proof The proof is by contradiction. Suppose there exists an $n$ such that $n>r+1$ and $T C(n, N)$ $<\mathrm{TC}(\mathrm{r}, \mathrm{N})$. Then the following inequalities hold:

$$
\text { i) } \frac{C_{p}+h(r+1, N)}{L(r+1)}>\frac{C_{p}+h(r, N)}{L(r)} \text {; and ii) } \frac{C_{p}+h(r, N)}{L(r)}>\frac{C_{p}+h(n, N)}{L(n)} \text {. }
$$

From equations (3.1), (3.4) and (3.6), we obtain:

$$
\frac{h(r+1, N)-h(r, N)}{L(r+1)-L(r)}>\mathrm{TC}(r, N)
$$

Similarly, from (3.1), (3.4) and (3.6), we also obtain:

$$
T C(n, N)=\frac{C_{p}+h(n, N)}{L(n)}>\frac{h(n, N)-h(r+1, N)}{L(n)-L(r+1)} .
$$

From (3.7), (3.8) and the assumption that $\mathrm{TC}(\mathrm{n}, \mathrm{N})<\mathrm{TC}(\mathrm{r}, \mathrm{N})$, we get:

$$
\frac{h(r+1, N)-h(r, N)}{L(r+1)-L(r)}>\frac{h(n, N)-h(r+1, N)}{L(n)-L(r+1)} .
$$


Noting that the numerator and the denominator are positive on both sides of the inequality given in (3.9), we can write:

$$
\frac{h(n, N)-h(r, N)}{L(n)-L(r)}>\frac{h(n, N)-h(r+1, N)}{L(n)-L(r+1)},
$$

which contradicts Lemma $3 b)$. Thus $\mathrm{TC}(\mathrm{n}, \mathrm{N})>\mathrm{TC}(\mathrm{r}, \mathrm{N})$ for all $\mathrm{n}>\mathrm{r}$.

Lemma 2, together with Theorem 1, thus produces an extremely simple, yet efficient procedure to obtain the optimal policy: sequentially evaluate $\mathrm{TC}(r, N)$, starting with $r=1$, until we encounter the first local minimum point. Due to the recursive form of $P_{f}(r, N), \tau(r, N)$ and $L(r)$, these values are computed very quickly from $P_{f}(r-1, N), \tau(r-1, N)$ and $L(r-1)$, all of which have been obtained at the previous step.

\subsection{Algorithm to find the optimal threshold value}

0 . Set $\mathrm{k}=1$. Compute $\mathrm{TC}(\mathrm{k}, \mathrm{N})$.

1. Set $k=k+1$. Compute $\mathrm{TC}(k, N)$.

2. If $\mathrm{TC}(\mathrm{k}, \mathrm{N})>\mathrm{TC}(\mathrm{k}-1, \mathrm{~N})$, stop; the optimal value of $\mathrm{r}$ is $\mathrm{k}-1$. Otherwise go to step 1 .

\section{Replacement at Failures}

In this section, we consider the situation in which failures of the system are detected at the instant they occur so that a failed system is immediately replaced by a new system. The model appears simpler in this case because there is no system-down cost incurred here. However, note that the computation of the expected cycle length is more complicated since it now depends on $\mathrm{N}$ as well as on $r$. Denote the expected cycle length as $L(r, N)$. Then, the expected cost incurred per unit time is

$$
\mathrm{TC}(\mathrm{r}, \mathrm{N})=\frac{\mathrm{C}_{\mathrm{p}}+\left(\mathrm{C}_{\mathrm{f}}-\mathrm{C}_{\mathrm{p}}\right) \mathrm{P}_{\mathrm{f}}(\mathrm{r}, \mathrm{N})}{\mathrm{L}(\mathrm{r}, \mathrm{N})} .
$$

In equation (4.1), the expression for $P_{f}(r, N)$ is given by equation (2.5). The expression for the term $\mathrm{L}(\mathrm{r}, \mathrm{N})$ is obtained by conditioning on the number of shocks which occur during the first inspection interval as

$$
L(r, N)=\sum_{j=0}^{r-1} q_{j}\left\{\bar{v}_{j}+L(r-j, N-j)\right\}+\sum_{j=r}^{N-1} q_{j} \bar{v}_{j}+\sum_{j=N}^{\infty} q_{j} \frac{N}{j+1} \bar{v}_{j} .
$$


Substituting the value for $\bar{v}_{j}$ given by equation (2.9) in equation (4.2), and collecting the terms involving $L(r, N)$, we obtain

with

$$
L(r, N)=\frac{1}{1-q_{0}}\left\{\frac{1}{\lambda} \sum_{j=0}^{N} j q_{j}+\frac{N}{\lambda} \sum_{j=N+1}^{\infty} q_{j}+\sum_{j=1}^{r-1} q_{j} L(r-j, N-j)\right\},
$$

$$
L(1, k)=\frac{1}{1-q_{0}}\left\{\frac{1}{\lambda} \sum_{j=0}^{k} j q_{j}+\frac{k}{\lambda} \sum_{j=k+1}^{\infty} q_{j}\right\}, \quad \text { and } L(0, k)=0 \text {. }
$$

We now develop some new properties, which parallel the properties presented in Lemmas 1, 2 and 3. Again, these properties, stated as Lemmas 4, 5, and 6, both reduce the amount of computation to obtain the term $\mathrm{L}(\mathrm{r}, \mathrm{N})$, and are used to prove the important characteristic of the cost function $\mathrm{TC}(\mathrm{r}, \mathrm{N})$ based on which the algorithm is devised.

Lemma 4. Let $\widetilde{A}(r, N)=\frac{P_{f}(r, N)-P_{f}(r-1, N)}{L(r, N)-L(r-1, N)}$. Then $\tilde{A}(r, N)=\widetilde{A}(r-n, N-n)$, for $1 \leq n \leq r-1$.

Proof The proof is similar to the proof of Lemma 2.

Lemma 5. $L(r, N)$ is increasing in $r$ for a given value of $N$.

Proof From Lemma 4, we obtain

$$
L(r, N)-L(r-1, N)=\frac{L(1, N-r+1)}{P_{f}(1, N-r+1)}\left\{P_{f}(r, N)-P_{f}(r-1, N)\right\}
$$

From property 1 , we know that $P_{f}(r, N)$ is increasing in $r$ for a given value of $N$. Thus, $L(r, N)-$ $\mathrm{L}(\mathrm{r}-1, \mathrm{~N})>0$.

Lemma 6. $\quad \frac{P_{f}(n, N)-P_{f}(r, N)}{L(n, N)-L(r, N)}<\frac{P_{f}(n, N)-P_{f}(r+1, N)}{L(n, N)-L(r+1, N)}$, for $n>r+1$.

Proof We first show that $\widetilde{\mathrm{A}}(\mathrm{r}, \mathrm{N})$ is increasing in $\mathrm{r}$ for a given $\mathrm{N}$. From Lemma 4, we obtain

$$
\tilde{A}(r, N)=\tilde{A}(1, N-r+1)=\frac{P_{f}(1, N-r+1)}{L(1, N-r+1)}, \quad \text { and } \quad \tilde{A}(r, N-1)=\tilde{A}(1, N-r)=\frac{P_{f}(1, N-r)}{L(1, N-r)} .
$$

From equations (2.7) and (4.4), we obtain

$$
P_{f}(1, N-r)>P_{f}(1, N-r+1) \text { and } L(1, N-r)<L(1, N-r+1) \text {. }
$$


Applying these relationships to (4.6), we get $\widetilde{A}(r, N-1)>\widetilde{A}(r, N)$, i.e., $\widetilde{A}(r, N)$ is decreasing in $N$ for a given r. From Lemma 4, it therefore follows that $\widetilde{A}(r, N)=\widetilde{A}(r-1, N-1)>\widetilde{A}(r-1, N)$. Thus, $\widetilde{\mathrm{A}}(\mathrm{r}, \mathrm{N})$ is increasing in $\mathrm{r}$ for a given $\mathrm{N}$. Now, we can use arguments similar to those used in the proof of Lemma 3, to show that equation (4.5) holds.

Entirely analogous to the proof of Theorem 1, we can now prove that the first local minimum encountered in the process of evaluating $\mathrm{TC}(\mathrm{r}, \mathrm{N}), \mathrm{r}=1, \ldots, \mathrm{N}$, is the global minimum. Noting $\mathrm{TC}(\mathrm{r}, \mathrm{N})$ is a recursive function with respect to $\mathrm{r}$ as before, we can therefore use the algorithm described in section 3 to obtain the optimal threshold value $\mathrm{r}^{*}$.

\section{Conclusions}

We have introduced a new preventive replacement policy for a standby system which consists of $\mathrm{N}$ components. The policy is based on the information about the number of failed components at each inspection point. We first obtained an expression for the expected cost per unit time for a given threshold value $r$ and, using a characteristic of the cost function $\mathrm{TC}(\mathrm{r}, \mathrm{N})$, we developed an extremely efficient procedure to find the optimal threshold value $r^{*}$. We also considered the case in which a failed system is replaced with a new one at the instant the system fails. For both cases, we provided a proof that the algorithm finds the optimum threshold value. 


\section{Appendix}

\section{Proofs of Properties 1 through 5}

Property 1. $P_{f}(r, N)$ and $\tau(r, N)$ are increasing in $r$ for a given $N$.

Proof Since $\mathrm{L}(\mathrm{r})$ is increasing in $\mathrm{r}$, property 1 follows from equation (3.3).

Property 2. $P_{\mathrm{f}}(\mathrm{r}, \mathrm{N})$ and $\tau(\mathrm{r}, \mathrm{N})$ are decreasing in $\mathrm{N}$ for a given $\mathrm{r}$.

Proof We will prove that $P_{f}(r, N)$ is decreasing in $N$ for a given $r$ by induction on $r$. We omit the proof for the case of $\tau(r, N)$ since it is similar to the proof for $P_{f}(r, N)$.

i) For $r=1$, from equation (2.6) we have $P_{f}(1, N-1)-P_{f}(1, N)=\frac{q_{N}-1}{1-q_{0}}>0$.

ii) Suppose $P_{f}(r, N)<P_{f}(r, N-1)$ holds for $r \leq k-1$. We now prove that the property holds for $r=$ k. From equation (2.6), we have

$$
P_{f}(k, N-1)-P_{f}(k, N)=\frac{1}{1-q_{0}}\left[q_{N-1}+\sum_{j=1}^{k-1} q_{j}\left\{P_{f}(k-j, N-1-j)-P_{f}(k-j, N-j)\right\}\right]
$$

From the induction hypothesis, $P_{f}(k-j, N-1-j)-P_{f}(k-j, N-j) \geq 0$ for $j=1, \ldots, k-1$. Thus $P_{f}(k, N-1)$ $-P_{f}(k, N)>0$. So by the principle of mathematical induction, $P_{f}(r, N)<P_{f}(r, N-1)$.

Property 3. (a) $\mathrm{P}_{f}(\mathrm{r}, \mathrm{N})-\mathrm{P}_{\mathrm{f}}(\mathrm{r}-1, \mathrm{~N}) \leq \mathrm{P}_{\mathrm{f}}(\mathrm{r}, \mathrm{N}-1)-\mathrm{P}_{\mathrm{f}}(\mathrm{r}-1, \mathrm{~N}-1)$

(b) $\tau(\mathrm{r}, \mathrm{N})-\tau(\mathrm{r}-1, \mathrm{~N}) \leq \tau(\mathrm{r}, \mathrm{N}-1)-\tau(\mathrm{r}-1, \mathrm{~N}-1)$

Proof We will prove property $3 \mathrm{a}$ ) by induction on $\mathrm{r}$. The proof of property $3 \mathrm{~b}$ ) is similar to the proof for property $3 a$ ) and is omitted.

i) For $r=2$, from equation (2.6), we have

$$
\left\{P_{f}(2, N-1)-P_{f}(1, N-1)\right\}-\left\{P_{f}(2, N)-P_{f}(1, N)\right\}=\frac{q_{1}}{1-q_{0}}\left\{P_{f}(1, N-2)-P_{f}(1, N-1)\right\} .
$$

From property $2 \mathrm{a}$ ), the above term is greater than 0 . (Also note that, for $r=1,(A .1)$ is trivially proved from the fact that $P_{f}(0, k)=0$.) 
ii) Suppose (A.1) holds for $r \leq k-1$. We now prove that the property holds for $r=k$. From equation (2.6), we have

$$
\begin{aligned}
& \left\{P_{f}(k, N-1)-P_{f}(k-1, N-1)\right\}-\left\{P_{f}(k, N)-P_{f}(k-1, N)\right\} \\
& \quad=\sum_{j=1}^{k-2} \frac{q_{j}}{1-q_{0}}\left[\left(\left\{P_{f}(k-j, N-1-j)-P_{f}(k-j, N-j)\right\}-\left\{P_{f}(k-1-j, N-1-j)-P_{f}(k-1-j, N-j)\right\}\right)\right. \\
& \left.\quad+\frac{q_{k}-1}{1-q_{0}}\left\{P_{f}(1, N-k)-P_{f}(1, N-k+1)\right\}\right]
\end{aligned}
$$

From the induction hypothesis, $\left\{P_{f}(k-j, N-1-j)-P_{f}(k-j, N-j)\right\}-\left\{P_{f}(k-1-j, N-1-j)-P_{f}(k-1-j, N-\right.$ j) $\}>0$ for all $j=1$ to $k-2$. Also from property $2 a), P_{f}(1, N-k)-P_{f}(1, N-k+1)>0$.

Thus, $\left.\left\{\mathrm{P}_{\mathrm{f}}(\mathrm{k}, \mathrm{N}-1)-\mathrm{P}_{\mathrm{f}}(\mathrm{k}-1, \mathrm{~N}-1)\right\}-\mathrm{P}_{\mathrm{f}}(\mathrm{k}, \mathrm{N})-\mathrm{P}_{\mathrm{f}}(\mathrm{k}-1, \mathrm{~N})\right\}>0$. So by the principle of mathematical induction, property $3 a$ ) holds.

Property 4. $A(r, N)$ and $B(r, N)$ are increasing in $r$ for a given $N$.

Proof To prove that $A(r, N)$ is increasing in $r$ for a given $N$, we first need to show that $A(r, N)$ is decreasing in $\mathrm{N}$ for a given $\mathrm{r}$. This can be shown using property $3 \mathrm{a}$ ) as follows:

$$
A(r, N)=\frac{P_{f}(r, N)-P_{f}(r-1, N)}{L(r)-L(r-1)} \leq \frac{P_{f}(r, N-1)-P_{f}(r-1, N-1)}{L(r)-L(r-1)}=A(r, N-1)
$$

Now from Lemma 2a) and inequality (A.5), we have $A(r, N)=A(r-1, N-1) \geq A(r-1, N)$.

The proof for $B(r, N)$ is the same as that for $A(r, N)$.

\section{Property 5.}

$$
\frac{h(n, N)-h(n-1, N)}{L(n)-L(n-1)}>\frac{h(n-1, N)-h(n-2, N)}{L(n-1)-L(n-2)}>\frac{h(n-2, N)-h(n-3, N)}{L(n-2)-L(n-3)}>\ldots
$$

Proof From the definitions of $h(r, N), A(r, N)$ and $B(r, N)$, we have

$$
\frac{h(r, N)-h(r-1, N)}{L(r)-L(r-1)}=\left(C_{f}-C_{p}\right) A(r, N)+C_{d} B(r, N)
$$

Since both $A(r, N)$ and $B(r, N)$ are increasing in $r$ for a given $N$ and both $C_{f}-C_{p}$ and $C_{d}$ are positive, property 5 is true. 


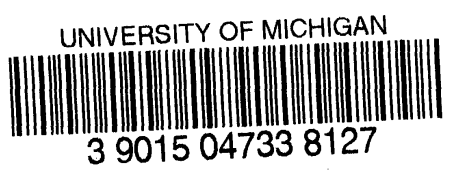

\section{References}

Barlow, R.E. and F. Proschan, Mathematical Theory of Reliability,Wiley,New York,(1965).

Gavish, B. and S.C. Graves, "A One Product Production/Inventory Problem Under Continuous Review Policy," Operations Research, v 28,(1980), pp. 1228-1236.

Gertsbakh, I.B. and G.L. Popov, "Optimum Choice of Preventive Maintenance Times for a Hierarchical System," Autom. Control Comp. Sci., No. 6(1972), pp. 24-30.

Heyman, D.P., "Optimal Operating Policies for M/G/1 Queueing Systems," Operations Research, v16 (1968), pp. 362-382.

Lee, H.S. and M.M. Srinivasan, "Control Policies for the $M \mathrm{X} / \mathrm{G} / 1$ Queueing Systems," Management Science, v 35 (1989), pp. 708-721.

Meine, H. and T. Asakura, "The Effect of an Age Replacement to a Standby Redundant System," J. Appl. Prob., V6(1969), pp. 516-523.

Ross, S.M., Applied Probability Models with Optimization Applications, Holden-Day Inc., (1970).

Sherif, Y. and M.L. Smith, "Optimal Maintenance Models for Systems Subject to Failure - A review," Naval Research Logistics Quarterly, v 28 (1981), pp. 47-74.

Srinivasan, M.M. and H.S. Lee, "Random Review Production/Inventory Systems with Compound Poisson Demands and Arbitrary Processing Times," to appear in Management Science, (1991). 